\title{
Prospects for Type Ia Supernova explosion mechanism identification with $\gamma$-rays
}

\author{
Jordi Gómez-Gomar ${ }^{1}$, Jordi Isern ${ }^{1}$ \\ Pierre Jean ${ }^{2}$ \\ ${ }^{1}$ Institut d'Estudis Espacials de Catalunya (IEEC), CSIC, Barcelona, Gran Capità 2 - 4, C.P. 08034, Spain. \\ ${ }^{2}$ CESR (CNRS), av. du Colonel Roche, BP 4346, 31028 Toulouse Cedex, France.
}

Accepted 31 August 2018. Received 31 August 2018; in original 31 August 2018

\begin{abstract}
The explosion mechanism associated with thermonuclear supernovae (SNIa) is still a matter of debate. There is a wide agreement that high amounts of of radioactive nuclei are produced during these events and they are expected to be strong $\gamma$-ray emitters. In the past, several authors have investigated the use of this $\gamma$-ray emission as a diagnostic tool. In this paper we have done a complete study of the $\gamma$-ray spectra associated with all the different scenarios currently proposed. This includes detonation, delayed detonation, deflagration and the off-center detonation. We have performed accurate simulations for this complete set of models in order to determine the most promising spectral features that could be used to discriminate among the different models. Our study is not limited to qualitative arguments. Instead, we have quantified the differences among the spectra and established distance limits for their detection. The calculations have been performed considering the best current response estimations of the SPI and IBIS instruments aboard INTEGRAL in such a way that our results can be used as a guideline to evaluate the capabilities of INTEGRAL in the study of type Ia supernovae. For the purpose of completeness we have also investigated the nuclear excitation and spallation reactions as a possible secondary source of $\gamma$-rays present in some supernova scenarios. We conclude that this mechanism can be neglected due to its small contribution.
\end{abstract}

Key words: $\gamma$-rays: general - supernovae: general.

\section{INTRODUCTION}

It is commonly accepted that Type Ia supernovae are the result of the thermonuclear explosion of a mass accreting $\mathrm{CO}$ white dwarf. In the outburst, high amounts of ${ }^{56} \mathrm{Ni}$ and other radioactive isotopes are produced opening the possibility to use $\gamma$-rays as a diagnostic tool. Although there is an agreement about the basic properties of such supernovae, there are several theories to account for these events. One class of models assumes that the parent white dwarf is very close to the Chandrasekhar mass and that the thermonuclear runaway starts at the center. According to the properties of the burning front, three cases can be considered: detonation model (Arnett 1969), deflagration model (Nomoto et al. 1984) and delayed detonation model (Khokhlov 1991). Recently, a fourth model based on a sub-Chandrasekhar mass progenitor has gained popularity due to some observational evidences (Phillips 1993; Maza et al. 1994; Hamuy et al. 1995). In this case, the explosion is triggered by the ignition of a freshly accreted He mantle (Ruiz-Lapuente et al. 1993; Livne et al. 1993; Wossley et al. 1994; Arnett 1994).
Regarding the amount of radioactive material synthesized, the chemical composition and the density and velocity profiles, the properties of ejecta are different for each one of these models. These differences affect the evolution of the total intensity of the $\gamma$-ray lines, their relative ratios and even their widths and shapes as well as the importance and extension of the continuum component of the spectrum.

Several authors have already investigated the $\gamma$-ray emission of type Ia supernovae (Gehrels et al. 1987; Ambwani \& Burrows 1988; Burrows \& The 1990, Burrows 1991; The et al. 1993; Ruiz-Lapuente et al. 1993b; Höflich \& Khoklov 1994; Kumagai \& Nomoto 1995; Woosley \& Timmes 1996) for different SNIa models. In most of these works a reduced number of SNeIa scenarios were studied, while in none of them a detailed determination of the detectability by INTEGRAL was performed. The aim of this paper is to accurately compute the evolution of the $\gamma$-ray spectra for a complete set of models, covering all the theories already mentioned, and to determine which spectral features could provide interesting information about SNIa. A Monte- 
Carlo $\gamma$-ray transfer code has been developed to compute the $\gamma$-ray emission for all the explosion models. Afterwards, the spectra have been convolved with the expected instrumental response for IBIS (Lei 1995) and SPI Jean et al. 1995) on-board of INTEGRAL to obtain the observational properties. A set of quantities that characterize the detectable spectral properties including line and continuum intensities and line shapes have been determined. We have also computed which are the detectability limits of these properties and investigated when, any given model could be rejected or identified if a SNIa is observed. Although the radioactive decay of freshly synthesized nuclei is the main source of $\gamma$ ray emission for SNIa, it is not the unique one. We have also investigated the emission produced by the nuclear excitation due to the interaction between the fast ejecta and the circumstellar medium, as would happen in the case of the explosion of a type Ia supernova in a symbiotic binary, or in a ISM cloud. Although, this mechanism is much weaker it has the advantage that it operates on longer time-scales (up to 1000's of years).

\section{MODELS AND RESULTS}

In order to compute the $\gamma$-ray spectra of the different models we have developed a code for the treatment of the $\gamma$-ray transfer, as described by Pozdnyakov et al. (1983) and Ambwani et al. (1988). It is based on the Monte-Carlo method technique, which allows the treatment of the comptonization process without approximations. With the code we can simulate the $\gamma$-ray spectra emitted by a SNIa with arbitrary composition, velocity and density profiles. Although many radioactive chains are included in the code only the following ones are important in the case of type Ia SNe:

$$
\begin{aligned}
& { }^{56} \mathrm{Ni} \rightarrow{ }^{56} \mathrm{Co} \rightarrow{ }^{56} \mathrm{Fe} \\
& { }^{57} \mathrm{Ni} \rightarrow{ }^{57} \mathrm{Co} \rightarrow{ }^{57} \mathrm{Fe}
\end{aligned}
$$

Three different sources of opacity have been taken into account: Compton scattering, photo-electric absorption and $\mathrm{e}^{+}$ $\mathrm{e}^{-}$pair production. The cross section for Compton scattering is given by the habitual Klein-Nishina expression, while absorption and pair production cross sections were taken from the compilation of experimentally evaluated data maintained by the Brookhaven National Laboratory

Three sources of $\gamma$ photons are considered besides nuclear decay: direct emission of two photons $(511 \mathrm{keV})$ by electron positron annihilation, indirect emission of two or three photons by positronium annihilation (Ore \& Powell 1949), and emission of fluorescence photons (not relevant in the present work since they are low energy photons $\mathrm{E}<10$ $\mathrm{keV}$ ). Simulations have been carried out to obtain both the evolution of the intensity of the strongest lines in the form of light curves and the detailed spectra at given times.

The properties of the ejecta for the different models have been kindly provided by E. Bravo who obtained them from accurate simulations of SNIa explosions. All the cal ulations were performed following the evolution of the system through the accretion phase and starting with a $0.8 \mathrm{~N} \odot$ partially cooled white dwarf with a composition $\left(\mathrm{X}_{C}=0.51, \mathrm{X}_{O}=0.49\right)$. The general procedure followed in the simulations is fully described in Bravo et al. (1996)

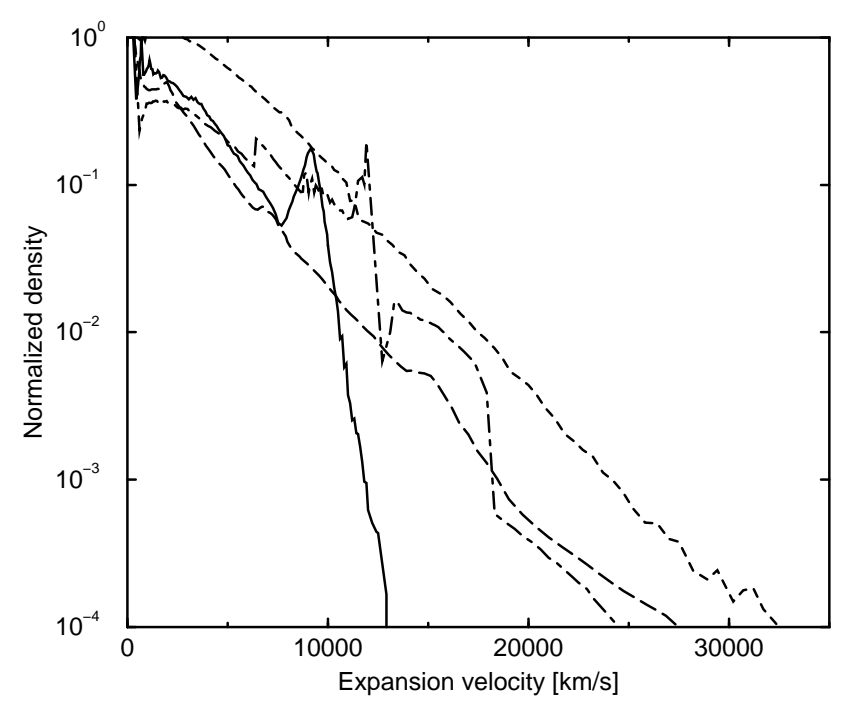

Figure 1. Density profiles as a function of the velocity of the ejecta for different models during the homologous expansion phase. Solid line corresponds to DEF model, long-dashed line to DEL model, dashed line to DET model and dot-dashed line to SUB model.

with the exception of the sub-Chandrasekhar model (hereafter,SUB) were a particularly accurate simulation of the accretion phase was carried out by José (José 1996) with a hydrodynamical code. This calculation is, up to date, the most consistent simulation of a sub-Chandrasekhar supernova in 1D. Three other models have been considered: DEF, DEL and DET, representing deflagration, delayed detonation and detonation supernovae, respectively. The details of the parameterizations adopted in the propagation of the burning front for DEF, DEL, and DET models are also described in Bravo et al. 1996. The main properties of these models at the beginning of the homologous expansion phase are summarized in Table 1 and Figure 1. The values in the table correspond to the ${ }^{56} \mathrm{Ni}$ and ${ }^{57} \mathrm{Ni}$ contain, mass of $\mathrm{C}+\mathrm{O}$, velocity of the shell with $\mathrm{m}=1 \mathrm{M}_{\odot}$ and total kinetic energy. The basic properties of our models (particularly, the ejected ${ }^{56} \mathrm{Ni}$ mass and kinetic energy) are compatible within uncertainities with those found in the literature (see for example models cdtg7 (Woosley \& Weaver1986) DF1 and DET1 (Höflich \& Khoklov 1996), N21 (Khokhlov 1991), Model 7 (Woosley \& Weaver 1994)).

The evolution of the $\gamma$-ray emission of these models is shown by the instantaneous spectra appearing in Figure 8 and by the light curves of the strongest lines (Figure 3). As expected from the models considered here the main properties of these spectra and light curves are compatible with those found in the literature for similar scenarios (see results for W7 (Burrows and The 1990) and DEF (Höflich et al. 1994); WDD2 (Kumagai \& Nomoto 1995) and N21 (Höflich et al. 1994); DET1(Höflich et al. 1994); Model 2 (Woosley \& Timmes 1996)). However, not all the properties are comparable since in some of these works either the continuum properties or the line profiles are not described. 
Table 1. Main properties of the ejecta after the beginning of the homologous expansion phase.

$\begin{array}{lcccccc}\text { Model } & { }^{56} \mathrm{Ni}\left[\mathrm{M}_{\odot}\right] & { }^{57} \mathrm{Ni}\left[\mathrm{M}_{\odot}\right] & \mathrm{C}+\mathrm{O}\left[\mathrm{M}_{\odot}\right] & \mathrm{v}(1 & \left.\mathrm{M}_{\odot}\right)\left[10^{9} \mathrm{~cm} / \mathrm{s}\right] & \mathrm{E}_{k}\left[10^{51} \text { ergs }\right] \\ \text { DEF } & 0.5 & 0.02 & 0.5 & 0.9 & 0.7 \\ \text { DEL } & 0.8 & 0.02 & 0.02 & 1.1 & 1.7 \\ \text { DET } & 0.7 & 0.04 & \sim 0 & 1.1 & 1.5 \\ \text { SUB } & 0.6 & 0.01 & 0.13 & 0.6 & 1\end{array}$

Twenty days after the explosion, the DEF model only shows a continuum component while the DEL, DET and SUB already display strong lines due to their higher expansion rates. Lines are particularly intense for DET and SUB models since they contain ${ }^{56} \mathrm{Ni}$ and ${ }^{56} \mathrm{Co}$ in the outermost shells. The efficiency of comptonization to produce continuum at low energies is limited in all models by the competing photo-electric absorption which imposes a cutoff below $40-100 \mathrm{keV}$. The energy of the cutoff is determined by the chemical composition of the external layers where most of the emergent continuum is formed at this epoch. In DEF and SUB models, comptonization mainly occurs in regions composed by intermediate-mass elements. As a consequence their continuum extends to lower energies than that of DET and DEL models since their comptonizing layers mostly contain Fe peak elements. Noticeable amounts of ${ }^{56} \mathrm{Ni}$ are still present at this epoch and the $158 \mathrm{keV}$ (Figure 2, top), 480 $\mathrm{keV}, 750 \mathrm{keV}$ and $812 \mathrm{keV}$ lines are really strong. The maximum intensities of these lines are very model dependent since at this moment the expansion rate and the distribution of ${ }^{56} \mathrm{Ni}$ strongly influence the spectrum. In models DEL and DET, the high expansion velocities of ${ }^{56} \mathrm{Ni}$ and ${ }^{56} \mathrm{Co}$ produce such broad lines that in some cases the lines emitted by both isotopes blend and lead to two peaked light curves (Figure 3, center).

Two months after the explosion(Figure 月, center), all the models ,except DEF, are close to their maximum luminosity (Figure 3). The ${ }^{56} \mathrm{Ni}$ lines have disappeared while the $122 \mathrm{keV}$ and $136 \mathrm{keV}$ lines produced by ${ }^{57} \mathrm{Co}$ are already visible although faint. At this moment, the line intensities in the DEL, DET and SUB models are mainly determined by the total mass of radioactive isotopes, while the effect of the expansion rate becomes secondary. The cutoff energy of DEL, DET and SUB models converge to a value of $\sim 70$ $\mathrm{keV}$ although that is still smaller for DEF.

Four months after the explosion the ejecta are optically thin in all models. The continuum is faint, and is dominated by a positronium annihilation component plus a contribution of photons scattered once. This contribution steeply decreases below $170 \mathrm{keV}$ (the energy of a backscattered $511 \mathrm{keV}$ ) and a step appears at this energy. During this phase, the cutoff is associated with the characteristic spectrum of photons emitted by positronium annihilations which is model-independent. Line intensities are now proportional to the mass of the respective parent isotopes.

Line profiles reveal during all their evolution differences in the velocity distribution of their parent isotopes. But at late epochs, when the models become transparent, these profiles give information about all layers of the ejecta (Figure 4. The lines corresponding to DET are the broadest ones and display a peculiar truncated core produced by the absence of ${ }^{56} \mathrm{Co}$ in the central layers, DEL and SUB lines show intermediate widths, while DEF lines are relatively narrow due to the low expansion velocity of its envelope. An interesting feature of the SUB lines is the presence of line wings which are the result of the typical "sandwich" distribution of ${ }^{56} \mathrm{Ni}$ in sub-Chandrasekhar SNeIa (see also, Woosley et al., 1996).

\section{DISCUSSION}

In the case of a close SNIa explosion $(\sim 1 \mathrm{Mpc})$ during the life time of INTEGRAL, high quality $\gamma$-ray spectra could be measured and detailed comparison between observations and theoretical results would be possible. However, when we consider more realistic distances $(>5 \mathrm{Mpc})$ the information provided by observations decreases drastically and only some outstanding features have a chance to be detected (Figure (). Our purpose is to determine which are these spectral features and what is the significance, if any, of the observational differences between models. To perform such study we have taken into account the theoretical spectra computed by us and the current estimations of the response of IBIS and SPI instruments included in the INTEGRAL mission (Lei 1995; Jean 1995). While SPI provides extremely good spectral capabilities appropriate for determining line properties, IBIS provides a higher effective area that makes it useful for broad band measurements of low energy continuum. It is important to note that the estimated sensitivity curves of these instruments do not include the instrumental sources of line background, which could be important at certain energies depending on the materials selected for the construction of INTEGRAL.

We have considered some quantities to summarize the properties of the spectra that are observable at relatively long distances and allow to easily determine differences between models. These properties are: line intensities, continuum intensities and line widths. In all calculations, an integration time of $10^{6}$ has been assumed.

\subsection{Line intensities}

Lines emitted by all the models are broader than SPI instrumental resolution. Therefore, the intensity of a line must be measured by integrating the flux in several instrumental channels forming a a band centered at the line energy. This implies on one side, that the effective sensitivity of SPI is reduced as compared to the narrow line case, and on the other side that it is important to appropriately select the band for the integration of counts to increase the significance of observations. In our calculations we have always adopted the band width which gives the measures with the maximum significance. This band varies not only with the line considered but also with the model. As a reference, in the case of a gaussian line the maximum significance is obtained for a 

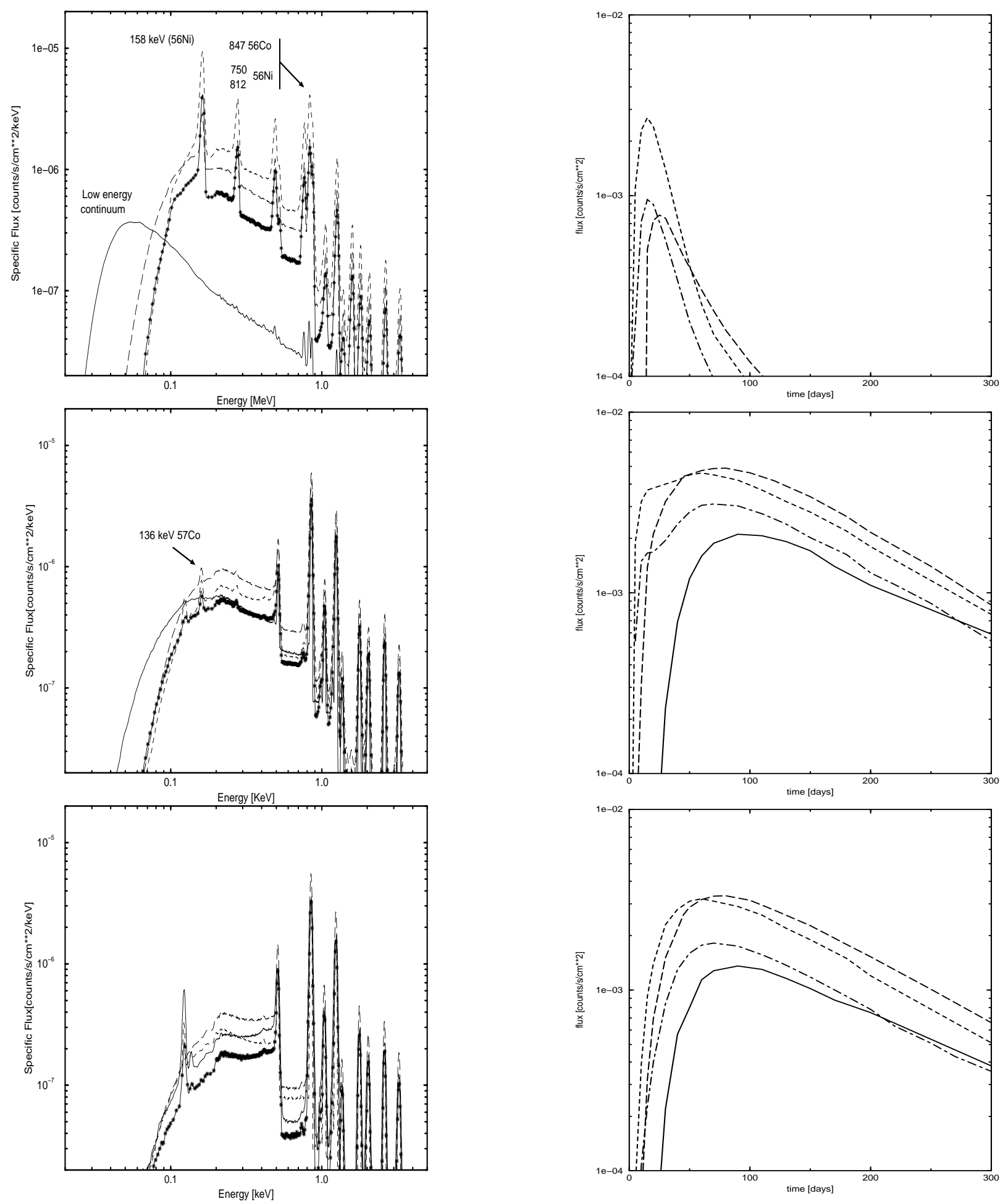

Figure 2. Spectral evolution for the four models $(5 \mathrm{Mpc})$ at 20 days (top panel), 60 days (middle panel) and 120 days (bottom panel) after the explosion. Solid lines correspond to DEF model, long-dashed lines to DEL model, dashed lines to DET model and starred-line to SUB model.

Figure 3. Light curves for the strongest lines assuming a distance of $1 \mathrm{Mpc}$. Top panel corresponds to the $158 \mathrm{keV}$ line, the central panel to the $847 \mathrm{keV}$ line and the bottom panel to the $1238 \mathrm{keV}$ line. Solid lines correspond to DEF model, long-dashed lines to DEL model, dashed lines to DET model and dot-dashed lines to SUB model. 


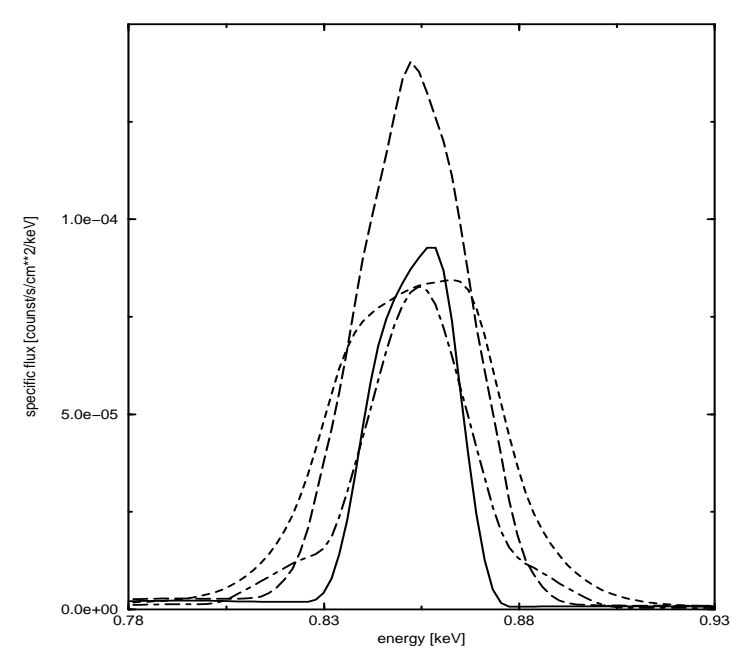

Figure 4. The profiles for $847 \mathrm{keV}$ line at 120 days. A distance of $1 \mathrm{Mpc}$ is assumed. Lines have the same meaning as in the previous figures.

bandwidth of $\sim 1.2$ FWHM (full width half maximum) and the number of counts contained in the band are $\sim 80 \%$ of the total line flux.

In our models, the lines with the highest intensities are: $511 \mathrm{keV}, 847 \mathrm{keV}$ (Figure 3, center), $1238 \mathrm{keV}$ (Figure 3, bottom) $812 \mathrm{keV}$ and $158 \mathrm{keV}$ (Figure 3, top). Table 2 summarizes the maximum distances at which the $158 \mathrm{keV}, 847$ $\mathrm{keV}$ and $1238 \mathrm{keV}$ lines can be detected. At these distances, the lines would be detected with a $3 \sigma$. Values were computed using the average flux around the time of maximum. In the table also appears the average number of the source counts detected in the case of an explosion at $5 \mathrm{Mpc}$. In all cases, this number falls far below the background level. In the table we can also see that the similar fluxes for DET and DEL lines correspond to different maximum detection distances due to the effect of the line widths in the sensitivity. In all cases, the strongest line is $847 \mathrm{keV}$ which is detectable up to distances between 11 and $16 \mathrm{Mpc}$. These distances are noticeably more pesimistic than those obtained by Kumagai et al. 1995 (distances from 40 to $56 \mathrm{Mpc}$ ) this is probably due to the fact that the broadeness effect has been included in our line sensitivity calculations for SPI. The differences in the fluxes measured at maximum range a factor of 2 between the different models. DET and DEL models display the highest fluxes, which are almost identical. In the case of DEF and SUB models, the fluxes are much lower. Similar relationships are found for the fainter $1238 \mathrm{keV}$ line, although in this case the fluxes are lower.

In contrast with Burrows (Burrows and The 1990), we have preferred to consider the $158 \mathrm{keV}$ line instead of the 812 $\mathrm{keV}$ line (both coming from the decay of ${ }^{56} \mathrm{Ni}$ ) because despite of being fainter its narrower profile makes it detectable at longer distances for SPI. The behavior of this line is very model-dependent. Despite being fainter than the $847 \mathrm{keV}$ one, $158 \mathrm{keV}$ is interesting in order to discriminate among
Table 3. $F(847)_{200 \text { days }} / F(158)_{\max }$

$\begin{array}{lc}\text { MODEL } & \text { ratio } \\ \text { DEF } & 8 \\ \text { DEL } & 2.2 \\ \text { DET } & 0.7 \\ \text { SUB } & 1.3\end{array}$

In the coefficients $\mathrm{F}(847)$ is taken at 200 days and $\mathrm{F}(158)$ at maximum.

models. This line is almost undetectable for the DEF model while for the SUB and DEL models it shows similar intensities and it is detectable up to distances of $\sim 7 \mathrm{Mpc}$. Finally, for a DET model, the $158 \mathrm{keV}$ line is even stronger than the $1238 \mathrm{keV}$ one, allowing its detection up to $12 \mathrm{Mpc}$.

An interesting coefficient is the relation between the 847 $\mathrm{keV}$ and the $158 \mathrm{keV}$ line fluxes $F(847)_{200 \text { days }} / F(158)_{\max }$ (see Table 3). This value provides information about the ratio between the total ${ }^{56} \mathrm{Ni}$ in the ejecta and the abundance of this isotope in the external layers. The late emission of $847 \mathrm{keV}$ is a consequence of the contribution of all ${ }^{56} \mathrm{Co}$ in the ejecta (produced mostly by the decay of ${ }^{56} \mathrm{Ni}$ ) while only the ${ }^{56} \mathrm{Ni}$ present in the outermost shells is responsible for the flux in the $158 \mathrm{keV}$ line.

\subsection{Continuum}

The properties of the continuum can be measured by obtaining the flux in fixed bands. In this, case the bands are broader than those used when measuring lines since the specific flux is lower. This means that one cannot expect to measure properties like spectral indexes or the position of the cutoff for continuum, but just fluxes in coarse channels. We have selected four bands taking into account the properties of the spectra and the detectability requirements. The 80-135 keV and 200-540 keV bands for SPI and the 40-80 $\mathrm{keV}$ and $80-135 \mathrm{keV}$ bands for IBIS.

Bands $40-80 \mathrm{keV}$ and $80-135 \mathrm{keV}$ contain information about the position of the continuum cutoff of the models (which is located in the interval $40-100 \mathrm{keV}$ ). At these energies the sensitivity of SPI degrades steeply preventing its use to detect the continuum below $80 \mathrm{keV}$ where only IBIS is considered.

The 200-540 keV band contains most of the emission produced by positronium annihilation which is the main source of continuum photons at late times. Due to the fact that emission of $511 \mathrm{keV}$ photons occur only in $25 \%$ of positronium annihilations, the significance of the flux in this band is higher than that for the $511 \mathrm{keV}$ line itself (see Table 4).

It is important to note that, when measuring flux in broad bands, the response of the instrument vary with the energy. To estimate the significance of the continuum measurements it is necessary to convolve the incident flux for each model with the instrumental exposure function and to compare the measured counts with the total background in the band. In this way, significances for the total flux in the band are obtained. The maximum distances at which the different models can be detected and the average number of source counts expected at $5 \mathrm{Mpc}$ are presented in Table 4. As for line intensities, fluxes have been averaged over a period of $10^{6} \mathrm{~s}$. The integration time is centered at 30 days 
Table 2. Intensities of the strongest lines.

\begin{tabular}{|c|c|c|c|c|c|c|c|c|c|c|c|}
\hline & & & $158 \mathrm{keV}$ & & $847 / 1238 \mathrm{keV}$ & & $847 \mathrm{ke}$ & & & $1238 \mathrm{k}$ & \\
\hline MODEL & $\mathrm{t}_{m}$ & d & $\mathrm{c}$ & $\mathrm{f}$ & $\mathrm{t}_{m}$ & d & c & $\mathrm{f}$ & d & $\mathrm{c}$ & $\mathrm{f}$ \\
\hline $\mathrm{DEF}$ & - & - & - & - & 95 & 11.2 & $610^{3}$ & $9.210^{-5}$ & 7 & $3.510^{3}$ & $5.210^{-5}$ \\
\hline DEL & 25 & 7 & $610^{3}$ & $3.110^{-5}$ & 75 & 16.2 & $1.310^{4}$ & $1.910^{-4}$ & 12.2 & $8.410^{3}$ & $1.310^{-4}$ \\
\hline DET & 14 & 12 & $210^{4}$ & $1.010^{-4}$ & 60 & 15.2 & $1.310^{4}$ & $1.810^{-4}$ & 10.6 & $7.710^{3}$ & $1.210^{-4}$ \\
\hline SUB & 16 & 8 & $710^{3}$ & $3.810^{-5}$ & 70 & 13.2 & $8.610^{3}$ & $1.210^{-4}$ & 9 & $4.610^{3}$ & $7.210^{-}$ \\
\hline
\end{tabular}

Time of maximum flux $\left(t_{m}\right)$ in days, maximum distance of detection $(d)$ in Mpc number of counts at $5 \mathrm{Mpc}(c)$ and flux at $5 \mathrm{Mpc}(f)$ for the strongest lines.

after the explosion, when the continuum is at maximum. At low energies the highest significances are obtained for the 80-135 keV band, although in this band differences among the models only range $\sim 30 \%$, being DEF and DET those which have the highest and the lowest fluxes respectively. Differences between models regarding flux in the $40-80$ $\mathrm{keV}$ band are really important. The DEF model is the only one that can be detected at these energies for distances $>$ $1 \mathrm{Mpc}$. The significances in this band for DEF are $\sim 20$ times higher than those for any other model and make it detectable up to $4 \mathrm{Mpc}$.

Significances for the $200-540 \mathrm{keV}$ band range a factor of 4 between the model with lowest fluxes (DEF) and that with the strongest emission (DET). The band can be detected up to $11 \mathrm{Mpc}$ for $\mathrm{DET}$ and is the best candidate for the observation of the $\gamma$-ray continuum in SNeIa. However, it does not provide with any information about the position of the cutoff an thus about the elemental composition of the ejecta.

\subsection{Line widths}

In principle, the good spectral resolution of SPI should allow to identify many details of the line profiles of SNeIa (Figure (4) and to perform model-specific fits to the observations. However, at distances larger than $\sim 2 \mathrm{Mpc}$ the fluctuations of the background hide the secondary features of the lines, (see Figure and in the majority of cases it is only possible to fit gaussians. At these distances, the width of the lines is the basic property of their profiles that we can measure. Although for DEF, DEL, DET and SUB models lines are not exactly gaussians, the difference between the FWHM of their theoretical profiles and that of the corresponding gaussian fit is, in all cases, below $\sim 3 \%$. These errors are negligible compared with observational uncertainties and hence gaussian fitting is a good technique to measure observational line widths. In what follows, we take the width of a line as the FWHM of its gaussian fit.

Our purpose is to estimate the maximum distances at which it is possible to obtain significant width measurements for the $\gamma$-ray lines predicted in our simulations. To do this, it is necessary to determine the errors associated to the observational fits. In opposition to what happens with measures of line intensities and continuum fluxes, the errors expected for widths cannot be analytically inferred from the instrumental properties since the average deviations of width measurements have complex dependencies on the properties of background fluctuations. To cricumvent this difficulty we have applied a bootstrap method by simulating an statistically significant number of "observations" of the lines emitted by the different models, placed at several explosion distances, in order to directly determine the distribution of the errors which appear when fitting their widths.

The procedure we apply is the following one. Taking a line and a explosion distance, the theoretical line profile at that distance is convolved with the exposure function of SPI (this function expresses the efficiency of the instrument to detect source photons) and with a gaussian that accounts for the limited resolution of SPI. The result of these two calculations is the signal that would be generated by the source line on the instrument in total counts per channel. However, in actual observations the source signal is additionally contaminated by the fluctuations due to the background subtraction procedure whose influence can only be predicted in a statistic way. To evaluate such effect, we have generated for each convolved line a set of hypothetical observations where random background fluctuations have been added to the source signal. These fluctuations are generated with a Monte Carlo procedure that takes into account the background properties predicted for SPI. A gaussian has been fitted to every "observation" by using the $\chi^{2}$ technique as it would be actually done in a real observation and finally a line width has been obtained. In each set more than 300 observations have been simulated.

With this procedure it is possible to determine the distribution of errors associated to the measurement of the width for any line, for any model and explosion distance. Calculations have only been performed for the $847 \mathrm{keV}$ line, since it has the maximum chance of being detected. The observations were taken 120 days after the explosion. The results obtained are summarized in Figure 6. where for each model a pair of curves is displayed. At every explosion distance the pair defines an interval of possible measured widths which contains the values that would be obtained by $90 \%$ of observers measuring the same line at the same distance (90\% dispersion bar). In the figure it can be appreciated that the dispersion of the measures is 0 for an explosion at distance 0 but it steeply grows with the explosion distance, being larger for lines with low fluxes. This is particularly important for the SUB and DEF models, since they have the lowest luminosities. For all models the distribution of hypothetical measures is skewed. That is, the observations are not symmetrically spread around the original line width but there is a tendency to measure widths larger than the original values which are indicated in the figure. As the possible errors become more important the significance of a measure decreases. Hence, it is necessary to adopt a quantitative criterium which establishes the maximum distance at which a measurement of a line width has physical meaning. We take this distance at the point at which the width of the dispersion bar for a line equals its original width. Assuming this definition the distances are: $\sim 5.5 \mathrm{Mpc}, \sim 8 \mathrm{Mpc}$ 
Table 4. Properties of the continuum.

\begin{tabular}{lcccccccc} 
& \multicolumn{9}{c}{ IBIS } & \multicolumn{4}{c}{ SPI } \\
MODEL & $40-80 \mathrm{~d}$ & $40-80 \mathrm{c}$ & $80-135 \mathrm{~d}$ & $80-135 \mathrm{c}$ & $80-135 \mathrm{~d}$ & $80-135 \mathrm{c}$ & $200-540 \mathrm{~d}$ & $200-540 \mathrm{c}$ \\
DEF & 4.2 & $410^{4}$ & 4 & $5.810^{4}$ & 3.8 & $710^{3}$ & 5 & $610^{3}$ \\
DEL & $<0.7$ & $10^{3}$ & 3.7 & $4.810^{4}$ & 3.5 & $610^{4}$ & 10 & $2.410^{4}$ \\
DET & $<0.7$ & $10^{3}$ & 3.6 & $4.610^{4}$ & 3.3 & $5.710^{4}$ & 11 & $2.810^{4}$ \\
SUB & 1 & $2.510^{3}$ & 3.9 & $5.310^{4}$ & 3.6 & $6.510^{3}$ & 8 & $1.510^{4}$
\end{tabular}

Maximum distances of detection (d) and counts at $5 \mathrm{Mpc}(c)$ for different continuum bands. All bands are in $\mathrm{keV}$ and the distances in Mpc.

Table 5. Line width measurements.

$\begin{array}{lcccc}\text { MOD/MOD } & \text { DEF } & \text { DEL } & \text { DET } & \text { SUB } \\ \text { DEF } & - & 4.5 \mathrm{Mpc} & 6 \mathrm{Mpc} & 4 \mathrm{Mpc} \\ \text { DEL } & 4.5 \mathrm{Mpc} & - & 7 \mathrm{Mpc} & <1 \mathrm{Mpc} \\ \text { DET } & 6 \mathrm{Mpc} & 7 \mathrm{Mpc} & - & 5 \mathrm{Mpc} \\ \text { SUB } & 4 \mathrm{Mpc} & <1 \mathrm{Mpc} & 5 \mathrm{Mpc} & \end{array}$

Distances at which the line widths allow to discriminate different models with at least $90 \%$ probability.

$\sim 7.5 \mathrm{Mpc}$ and $\sim 6 \mathrm{Mpc}$ for DEF, DEL, DET and SUB respectively.

But more important than determining when the width of a line can be measured, is to know when this measure will be useful to discriminate among the different models. Figure 6 provides the information necessary to reject or identify models from observations using measured widths. Taking the measured width and the explosion distance for a given observation an observer can reject all the models whose associated pair of curves in the figure do not contain the measure. By doing this we ensure a probability $>90$ $\%$ of correct model rejection. In the same way, if the pair of curves corresponding to only one of the models contains the given measure, the observer can identify this model as observed. This would lead to a correct model identification in more than $90 \%$ of cases. However, the confidence of the method decreases when we consider distances where the 90 $\%$ dispersion bars of two or more models overlap. For any pair of models, the point of intersection between the respective pairs of curves places the distance below of which it can be assured that model discrimination with the use of witdh measurements will be correctly performed in more than 90 $\%$ of observations. We take this distance as the maximum distance reasonable for model discrimination by means of width measurements. In Table $\mathrm{B}$ we summarize these maximum distances for the different combinations of models. Line widths for SUB and DEL models are indistinguishable for distances larger than $1 \mathrm{Mpc}$. However, in the remaining cases the differences between each model pair are noticeable and it is possible to discriminate among them up to distances of the order of 4 to $7 \mathrm{Mpc}$. Particularly interesting are the differences between DEL and DET models which otherwise have very similar line intensities (Table 2).

If we had adopted a level of confidence lower than 90 $\%$ it would have been possible to define longer distances for model discrimination. However, due to the steep increase of the dispersion at the distances the limits obatined would not be appreciably longer.

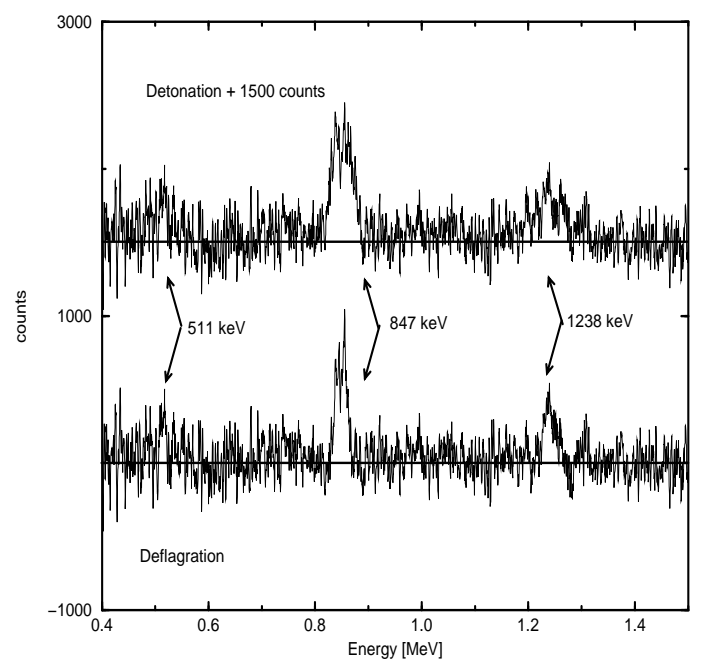

Figure 5. Simulated "observational" spectra for a detonation and a deflagration SNIa at $5 \mathrm{Mpc}$ (integration time $=10^{6} \mathrm{~s}$ ). The detonation spectrum is shifted a factor +1500 . Obtained by Jean et al. (1995b).

\section{NUCLEAR EXCITATION}

Radioactivity is not the only source of $\gamma$-ray emission in SNIa explosions. The interaction of the high velocity ejecta with the surroundings can induce emission of $\gamma$-rays due to nuclear excitations and spallation reactions. For comparison purposes with the radioactive decay mechanism, we have investigated the importance of such emission source in the case of SNeIa. Two situations have been considered: the interaction of the high velocity ejecta with the stellar wind emitted by the supernova progenitor (case of the explosion of a symbiotic binary, for instance) and the interaction with a relatively dense interstellar medium.

In the symbiotic binary scenario we have assumed a system formed by a red giant, a white dwarf and an associated stationary wind. We have adopted a total mass of $3 \mathrm{M}_{\odot}$, an orbital period of $1 \mathrm{yr}$ and a separation of $1.7 \mathrm{AU}$. The composition of the wind is taken to be solar while that of the ejecta is taken from the DEF, DEL, DET and SUB models respectively. If we assume an accretion efficiency of $10 \%$, an accretion rate appropriate for a sub-Chandrasekhar supernova $\left(\sim 10^{-8} \mathrm{M}_{\odot} \mathrm{yr}^{-1}\right)$ and a wind velocity of $10 \mathrm{~km} / \mathrm{s}$, 


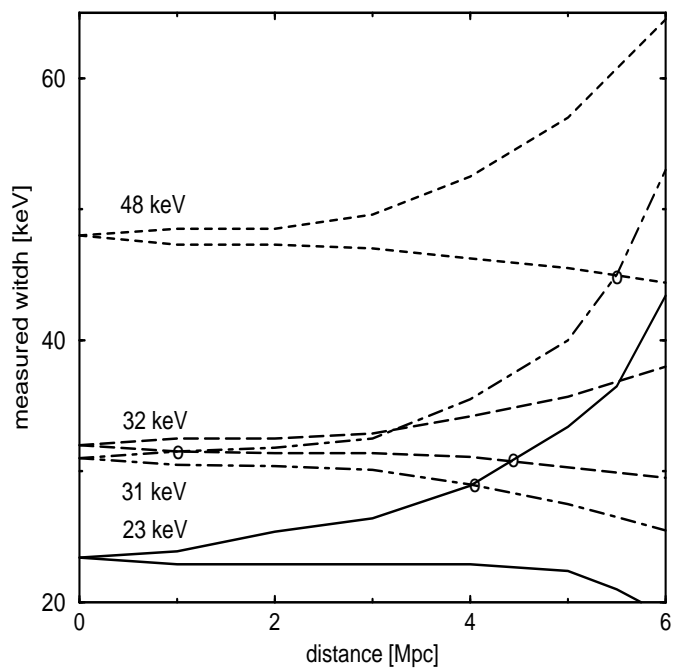

Figure 6. Pairs of curves containing $90 \%$ of observational line widths as a function of distance for the $847 \mathrm{keV}$ line (120 days after the explosion). The dispersion of the measures is due to the fluctuations of the background. Solid line corresponds to DEF model, dotted line to DEL model, dashed line to DET model and dot-dashed line to SUB model. Also indicated appears the original line width for each model.

the density profile of the wind is:

$$
\rho=\frac{10 \dot{M}_{a c c r}}{4 \pi r^{2} v_{\text {wind }}}=\frac{2.510^{11}}{r^{2}} \mathrm{gr} / \mathrm{cm}^{3}
$$

where $r$ is the distance to the center of the system. In this scenario we have not considered any energy losses of the ejecta since the $\gamma$-ray emission almost disappears after 10 15 yrs, much earlier than the beginning of the Sedov phase of the expansion.

In the case of an explosion in a ISM region, the density is a free parameter. The average density of the ISM in the galactic plane is $\sim 1 \mathrm{~cm}^{-3}$ but regions with densities ranging $10-100 \mathrm{~cm}^{-3}$ are not rare. In principle, SNeIa are not specifically associated with dense ISM regions but they could meet such regions when crossing the galactic plane. In this scenario, the ISM region is considered to be unbounded and hence the emission will be persistent. In this case, the effects of braking have to be taken in to account since they are the main responsible for the late decay of the light curve. They are caused by ionization and excitation of the ISM atoms and can be described by the typical Bethe-Block equation which takes into account the composition of the ejecta and of the ISM. In this scenario both, the intensity of the emission and the stopping power are proportional to the density of the medium and lead to the following scaling law for the light curves as a function of the ISM density:

$$
L(t, n)=\frac{n}{n_{0}} L\left(t \frac{n}{n_{0}}, n_{0}\right)
$$

where $\mathrm{n}$ and $\mathrm{n}_{0}$ are different ISM densities and $\mathrm{t}$ is the time.

We have computed the main light curves induced by the DEL, DET and SUB models in both scenarios. The DEF model does not eject mass with energies above the threshold of the excitation reactions and it can be ignored. The method used to compute the light curves is described in Fields et al. (1996). The basic equation expressing the intensity of a line (l) generated by the interaction of species (i) in the ejecta with the species (j) in the surrounding medium (with homogeneous composition) is:

$L_{l}=\frac{1}{4 \pi d^{2} A_{i} m_{p}^{2}} \int_{0}^{E_{\max }} n_{j}(E, t) \sigma_{i j}^{l}(E) X_{i}(E) \frac{d M}{d E} v(E) d E$

where the specific kinetic energy across the ejecta is taken as the integration variable, $\mathrm{d}$ is the distance to the observer $X_{i}$ is the mass fraction of species $\mathrm{i}, n_{j}$ is the number density of the $(\mathrm{j})$ species in the medium, $\mathrm{v}(\mathrm{E})$ is the velocity of a layer of the ejecta with specific kinetic energy $\mathrm{E}$ and $\sigma_{i j}^{l}(E)$ is the energy-dependent cross section of the excitation reaction. Contrary to what was done in Fields et al., in this work braking effects are included and thus the profile of the kinetic energy of the ejecta as a function of the mass coordinate evolves with time. Cross sections were taken from Ramaty et al.(1979) and include direct photon emission and de-excitation of spallation products.

The results of these calculations are summarized in Figures 7 and 8 (this figure is valid for all ISM densities due to the scaling law previously explained) where the main light curves are displayed. The isotopes responsible for the strongest lines are: DEL $\left({ }^{56} \mathrm{Fe},{ }^{28} \mathrm{Si},{ }^{40} \mathrm{Ca}\right)$, DET $\left({ }^{56} \mathrm{Fe},{ }^{28} \mathrm{Si}\right.$, $\left.{ }^{40} \mathrm{Ca},{ }^{32} \mathrm{~S},{ }^{12} \mathrm{C},{ }^{16} \mathrm{O}\right)$ and SUB $\left({ }^{56} \mathrm{Fe},{ }^{28} \mathrm{Si},{ }^{40} \mathrm{Ca}\right)$. The main conclusion is that the excitation mechanism is in general negligible. The emission is extremely weak, even when galactic supernovae are considered, and in all cases, the luminosity coming from the radioactive decay would overwhelm the excitation lines during at least the first ten years (Figure 7). In both scenarios the models with the strongest and the faintest emission are the DEL and SUB ones respectively.

In the symbiotic scenario, the strongest line (4439 keV), can be detected at a maximum distance of $1 \mathrm{kpc} 10$ years after the explosion and just 100 pc after 100 years. This means, that SNIa explosions in symbiotic systems can be ruled out as a source of detectable emission produced by nuclear excitation.

In the ISM scenario, the emission is also extremely low although the persistence of lines increases somewhat the chances of detection. If a ISM density of $100 \mathrm{~cm}^{-3}$ and the DEL model are considered, the $4439 \mathrm{keV}$ line shows a nearly constant intensity during 150 years before starting to decay. During this period, the line could be detected by INTEGRAL for explosions closer than $\sim 1.5 \mathrm{kpc}$. If we assume an ISM density of $10 \mathrm{~cm}^{-3} \mathrm{~cm}$, the line emission lasts for 1500 yrs but in this case it is only detectable at distances < 500 pc. Thus, nuclear excitation can be safely neglected in all cases in comparison with radioactive decay as a source of $\gamma$-rays in SNIa explosions. Only in the improbably case that one of the recent close SNeIa (SN 1006, SN Tycho) remmants was associated with a dense ISM region, its excitation emission could be detected by INTEGRAL.

\subsection{Conclusions}

The simulations performed here show clear differences between $\gamma$-ray spectra of deflagration, detonation, delayed detonation and sub-Chandrasekhar models of SNeIa. These dif- 


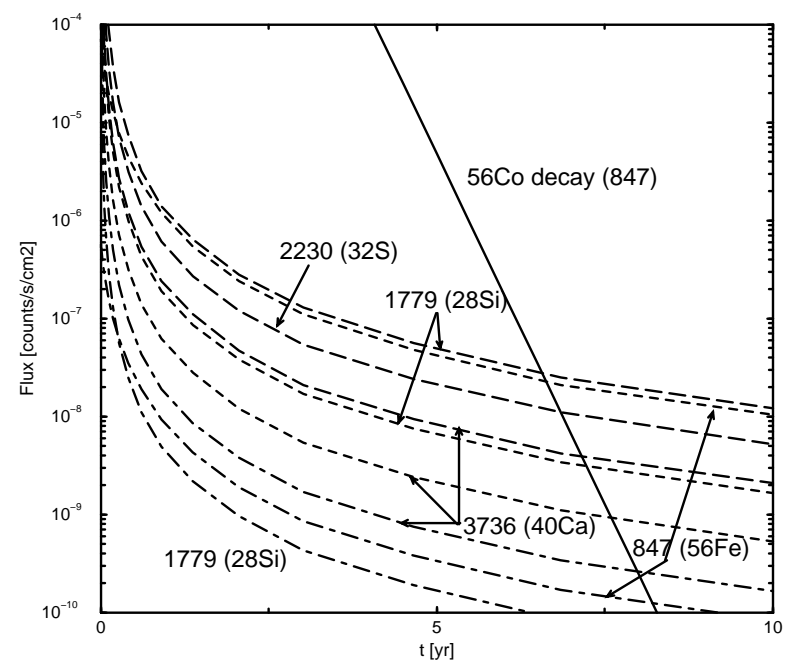

Figure 7. Evolution of the flux of the main excitation lines for a SNIa explosion in a symbiotic binary, compared with $847 \mathrm{keV}$ line produced by the decay of ${ }^{56} \mathrm{Co}(10 \mathrm{kpc})$. The lines have the same meaning as in previous figures.

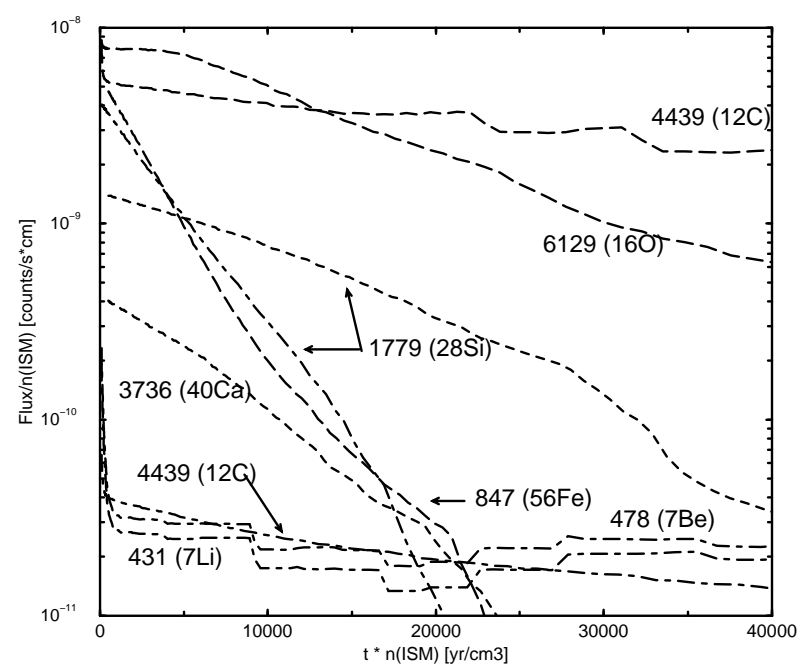

Figure 8. Evolution of the flux the main excitation lines produced by the explosion of a SNIa in a dense ISM (10 kpc). X and $\mathrm{Y}$ axes can be rescaled as a function of the ISM density. Lines have the same meaning as in previous figures.

ferences are particularly important at early times, when the total amount of radioactive isotopes and their distribution, expansion rate and composition of the ejecta affect the $\gamma$-ray emission. After the maximum, differences are only related with total mass of ${ }^{56} \mathrm{Co}$ and its velocity distribution. The main differences between the spectra are found in the in- tensity and profiles of the strongest lines coming both from ${ }^{56} \mathrm{Ni}$ and ${ }^{56} \mathrm{Co}$ decays, as well as in the intensity, shape and cutoff of the low energy continuum.

Since the level of background is high and the instrumental capabilities are limited, not all the differences present in the emergent spectra of the models are actually observable by an instrument like INTEGRAL. Due to the intrinsic width of SNIa lines, the effective sensitivity of SPI to detect them is reduced by a factor $\sim 3-4$ respect to the narrow line case. The strongest SNIa spectral feature is the $847 \mathrm{keV}$ line which can be measured with $3 \sigma$ for distances up to 11 to $16 \mathrm{Mpc}$. Important variations appear for the fainter 158 $\mathrm{keV}$ line which allow to discriminate models up to $6 \mathrm{Mpc}$. This line is detectable for the a detonation SNIa up to 11 Mpc. The continuum must be measured using broad bands. Emission in the $80-135 \mathrm{keV}$ could be observed $(3 \sigma)$ at 4 $\mathrm{Mpc}$ although in this case there are only small differences among the different models. The flux in the $40-80 \mathrm{keV}$ band is a peculiar feature of deflagration SNIa and could be observed with IBIS up to $4 \mathrm{Mpc}$. Concerning line profiles, in principle they can be resolved by SPI. However the limited sensitivity reduces the information that can be obtained about the shapes and only line widths can be measured at reasonable distances. We have shown here that it is possible to discriminate between any pair of models for distances up to $4-6 \mathrm{Mpc}$ by measuring the width of $847 \mathrm{keV}$ line except in the cases DEL and SUB.

From these results, the observation of a SNIa explosion is possible with INTEGRAL for any event closer than $11 \mathrm{Mpc}$. If the explosion mechanism of SNIa is a detonation they could be observed at distances as long as $16 \mathrm{Mpc}$. However, only if a SNIa explodes at a distance of $\sim 6 \mathrm{Mpc}$ or less, INTEGRAL could obtain enough information to univocally discriminate between the $\gamma$-ray emission patterns corresponding to DEF, DEL, DET and SUB models. In fact, only a single recent SNIa (SN1986G) fulfilled this requirement.

Finally, it has been shown that the nuclear excitation mechanism can be ruled out as a source of detectable $\gamma$ ray emission. In any of the proposed scenarios this emission is totally dominated by the radioactive decay emission for more than ten years. After that, in the symbiotic scenario the luminosity decays very quickly. Only a SNIa event that had happened in the solar neighborhood during the last few centuries and that was associated with a high density ISM could be detected as a fossil $\gamma$-ray emitter.

\section{ACKNOWLEDGMENTS}

We wish to thank Eduard Bravo and Jordi José, who provided us with initial models for our calculations. Peter von Ballmoos discussed with us fundamental questions about the instrumental response of SPI and gave us an "observational" perspective of the problem. This work has been financed by the projects: CICYT (ESP95-0091) and CIRIT (GRQ-8001).

\section{REFERENCES}

Ambwani K. and Sutherland R., 1988, ApJ 325, 820 
Arnett D., 1969, Ap.Space Sci. 5, 280

Arnett D., Livne E., 1994, ApJ 427, 315

Bravo E., Tornambé, Domínguez I. and Isern J., 1996 A\&A 306, 811

Evaluated photoatomic data base Brookhaven Center for Radiation Research, 1991

Burrows A., The L.S., 1990, ApJ 360,626

Burrows A., 1991 in Gamma-Ray Line Astrophysics: Proceedings of the Internanional Symposium, Paris, ed. Durochoux P., Prantzos N., (AIP Conference Proceedings: New York), 297

Bykov A. and Bloemen, A\&A 283,L1-L4

Clayton D.D., 1982 in Essays in Nuclear Astrophysics, ed. Barnes C.A., Clayton D.D. and Schramm D.N. (Cambridge: Cambirdge University Press)

Fields B.O., Casse M., Vangioni E. and Nomoto K., 1996, ApJ 462,267

Gehrels N., Leventhal M., MacCallum C.J., 1987 , ApJ 322, 215

Hamuy M., Phillips M.M., Maza S., Suntzeff N., Schommer R.A. and Aviles R., 1995, ApJ 109

Höflich P., Khokhlov A. and Müller E., 1994, ApJ Supplements 92,501

Höflich P., Khokhlov, 1996, ApJ 457, 500

Jean P., Naya, J.F., Olive, P. and Von Ballmoos, 1995, A\&A

Jean P. and Von Ballmoos, 1995, private communication

José, 1996 Ph.D. Thesis, Barcelona University

Khokhlov A.M., 1991, A\&A 245, 114

Kumagai S., Nomoto K., 1995 in Proceedings of the NATO ASI on Thermonuclear Supernovae, Aiguablava, Eds. Canal R., Ruiz-Lapuente P., Isern J.

Lei F., 1995, Efficiency Background and Sensitivity Estimates for IBIS, ESA Tech. Not.:IN-IM-SOT-TN-0024

Livne, E. and Arnett D., 1993, ApJ 415, L107

Maza J., Hamuy M., Phillips M.M., Suntzeff N.B. and Avileés R., 1994, ApJ Letters 424,L107

Munari U. and Renzini A., 1992, ApJ Letters 397, L87-L90

Niemeyer J.C., Hillebrandt W., 1995, ApJ 452,769

Nomoto K., 1980, in IAU-Symposium 93, ed. Sugimoto D., Lamb D.Q. and Schramm D., (Dordretch:Reidel)

Nomoto K., Thielemann F.K. and Yokoi K., 1984 , ApJ 286, 664

Ore A. and Powell J.L., 1949, Physical Review, Vol. 75, 11, 1696

Phillips M.M., 1993, ApJ Letters 413, L105

Pozdnyakov L.A., Sobol I.M., Syunyaev R.A., 1983, Soviet Scientific Reviews. Section E: Astrophysics and Space Physics Reviews, Vol 2, 184

Ramaty R., Kozlovsky B. and Lingenfelter R.E., 1979, ApJ Supplements 49, 487

Ramaty R., 1986, Nuclear Processes in Solar Flares in Physics of the Sun, ed. Sturrock, Holzer, Mihalas and Ulrich, (Kluwer Academic Press)

Ramaty R., Kozlovsky B. and Lingenfelter R., 1995, ApJ Letters 438, L21-L24

Ruiz-Lapuente P., Jeffery D., Challis P.M., Filippenko A.V., Kirhsner R.P., Ho L.H., Schmidt B.P., Sànchez F. and Canal R., Nature, 365, 728

Ruiz-Lapuente P., Lichti G.G., Lehoucq R., Canal R. and Cassé, 1993b, ApJ 417, 547

The L.S., Lesising D., Clayton D., ApJ, 403, 32

Tueller J., 1991 in Gamma-Ray Line Astrophysics: Proceedings of the Internanional Symposium, Paris, ed. Durochoux P., Prantzos N., (AIP Conference Proceedings: New York), 199

Whelan J. and Iben 1973,ApJ 186, 1007

Woosley S.E., Weaver T.A, 1986, in Radiation Hydrodynamics in Stars and Compact Objects ed. D. Mihalas and K.H. A. Winkler (New York: Springer-Verlag)

Woosley S.E.,1991 in Gamma-Ray Line Astrophysics: Proceedings of the Internanional Symposium, Paris, ed. Durochoux P., Prantzos N., (AIP Conference Proceedings: New York),
270

Woosley S.E. and Weaver T.A., 1994, ApJ 423, 371

Woosley S.E. and Timmes F., 1996, in Proceedings of the 2nd INTEGRAL Workshop, St.Malo, ed C. Winkler, in preparation Yungelson L., Livio M., Tutukov A., Kenyon S.J. 1995 ApJ 447, 656

(C) 0000 RAS, MNRAS 000, 110 